\title{
Pain and anxiety experiences of South African adult burn injury patients during physiotherapy management
}

\begin{abstract}
Adequate management of procedural pain during physiotherapy management plays an important role in building a trusting relationship between the burn victim and the physiotherapist, and in ensuring desirable functional outcomes. However, the burn pain management regimens currently utilized in burn units, primarily consist of traditional pharmacologic analgesics which are associated with numerous side-effects and alone are often reported as inadequate to alleviate procedural pain, warranting safer and effective adjunct therapies.

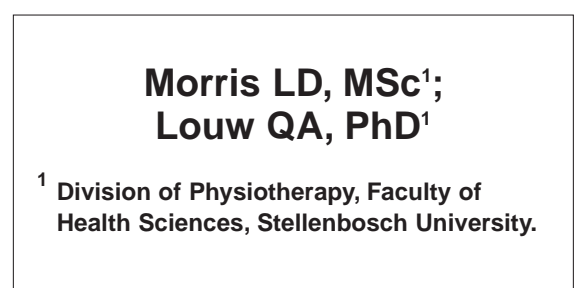
Prior to the introduction and implementation of adjunct therapies into a developing world, it is imperative that the current situation in a burn unit, in terms of whether or not the pain management regimens in place are adequate, is first assessed, due to cost concerns. The following short report exemplifies the pain and anxiety experiences of a small number of burn injury patients during physiotherapy at the Tygerberg Hospital adult burn unit, South Africa. It was hypothesized that the results of this study would underpin whether adult burn injury patients in a developing country require adjunct therapies during physiotherapy management to supplement traditional pharmacologic analgesics in managing their procedural pain and subsequent anxiety.
\end{abstract}

\section{KEYWORDS: BURN INJURY, ADULTS, PAIN, ANXIETY AND PHYSIOTHERAPY.}

\section{INTRODUCTION}

Successful participation in physiotherapy after a severe burn is crucial for minimizing long-term disability. It prevents the healing skin from contracting and losing its elasticity, which could potentially lead to a reduced range of limb motion and a decrease in function (Hoffman et al 2000). Unfortunately, rehabilitation for burn injury patients is hindered by pain and subsequent anxiety caused by the physiotherapy management itself and the anticipation of the treatment session. As a result, patients are often discouraged from being compliant during their treatment sessions which may lead to a decrease in functional outcomes (Hoffman et al 2000; van Twillert et al 2007).

\section{Correspondence to:}

Linzette Deidré Morris

Division of Physiotherapy

Faculty of Health Sciences

Stellenbosch University

PO Box 19063,

Tygerberg 7505

South Africa

Email: 1dmorris@sun.ac.za
However, the appropriate management of procedural pain in burn injury patients (pain felt during procedures such as physiotherapy management and wound dressing changes) has been a major challenge for health professionals working in burn units globally (Abdi et al 2002, Richardson et al 2009, ConnorBallard et al 2009). Concerns for the risk of drug addiction, drug side-effects, underestimation of the complexity of burn pain and ignorance regarding the proper administration of analgesics during burn rehabilitation often leads to the under treatment of burn pain by health professionals (Ptacek et al 1995; Jonsson et al 1998; Richardson et al 2009). This is of concern as uncontrolled burn pain could result in physical and psychosocial problems and destroy the trust patients may have in their health provider (Connor-Ballard et al 2009). Adequate management of procedural pain thus plays an important role in building a trusting relationship between the burn victim and the physiotherapist, in reducing subsequent anxiety and in promoting patient compliance with rehabilitation, thereby improving functional outcomes (de Jong et al 2007).
The current burn pain management regimens for procedural pain used in most burn units primarily consist of the administration of traditional pharmacologic analgesics, more specifically, opioids such as Morphine, which are associated with numerous side-effects. Alone these analgesics are often reported as inadequate to successfully alleviate procedural pain, warranting the use of adjunct therapies (Haik et al 2006; van Twillert et al 2007). Non-pharmacologic adjunct therapies usually pose less threat to the burn injury patient than traditional pharmacologic analgesics, as they are associated with less side-effects, are less addictive and less invasive (Hoffman et al 2006). Although resources in developed countries allow for the possibility of implementing expensive adjunct therapies such as distraction techniques and technologies; in developing countries, such as South Africa, this is not always possible as healthcare budgets are stringent (Louw et al 2007). It may therefore not be economically feasible to implement expensive interventions from first world countries into a third world clinical setting. 
For this reason it is imperative that the current burn pain management regimens in place in developing countries are first assessed to ascertain the need for adjunct therapies, before implementation takes place. The following short report thus exemplifies the pain and anxiety experiences of a small number of burn injury patients during physiotherapy at one of the major tertiary institutions for adult burn injuries in the Western Cape, South Africa. It was hypothesized that the results of this study would preliminarily underpin whether adult burn patients in a developing country require adjunct therapies during physiotherapy management to assist in managing their procedural pain and subsequent anxiety.

\section{METHODS}

Ethical approval for this study was granted by the Committee for Human Research at the Stellenbosch University. A month-long prospective survey was conducted at the Tygerberg Hospital's adult burn unit in South Africa during October 2008. Subjects were recruited consecutively if they were male or female, between the ages of 18 years and older, had sustained a burn injury of any degree and to any part of their body, and underwent physiotherapy burn management. Patients, whose medical condition was deemed unstable, were unconscious and unaware of their surroundings, and who had any cognitive deficits were excluded from the study. Informed consent was obtained from all subjects prior to participation in the study. Demographical, personal, burn injury, analgesic and physiotherapy treatment information, as well as baseline pain and anxiety data using the Numeric Pain Rating scale (NPRS) and the Burn Specific Pain and Anxiety scale (BSPAS) (Taal and Faber 1997), for each subject was extracted or collected, and recorded. (The BSPAS consists of nine items which describe feelings of worry, tension, fear and anxiety. The items are scored on $10 \mathrm{~cm}$ numeric pain rating scales with two reference points given values of 0 and 10. The values were spaced $1 \mathrm{~cm}$ apart (Taal and Faber 1997).
The maximum duration of the physiotherapy treatment session, which consisted of passive range of motion exercises in the same planes of movement, was 20 minutes and was divided into two equal components. Midway through and immediately after the physiotherapy session, a blinded measurer administered the second and third sets of the NPRS and BSPAS forms. Thirty minutes after the physiotherapy session, the blinded measurer administered the final set of the NPRS and BSPAS forms to the subject. In essence, four pain and anxiety scores were recorded: before the physiotherapy session, midway through the physiotherapy session, immediately after the physiotherapy session and 30 minutes after the physiotherapy session.

Student's paired t-tests were used to analyze differences between mean pain and anxiety scores before and during-; before and after-; and before and 30 minutes after the physiotherapy session (significant level <0.05). The t-distribution was used to calculate $95 \%$ confidence intervals around the mean differences. In addition, descriptive statistics incorporating the median and range were also done, to illustrate changes in severity of pain and anxiety during physiotherapy.

\section{RESULTS}

\section{Participants}

A total of 17 (13 males and 4 females) adult burn injury patients, with a median age of 33 years (range 20 to 56 years) admitted to the TBH adult burn unit, consented to participating in this phase of the study. Eight of the 17 subjects were

Table 1: Pain scores (mean and median scores) as reported by subjects before, during and after physiotherapy sessions.

\begin{tabular}{|l|l|l|}
\hline & \multicolumn{2}{|c|}{ Pain scores ( $\mathrm{n}=17)$} \\
\hline & Mean (SD) & Median (range) \\
\hline Before physiotherapy & $4.4(2.9)$ & $5(0-8)$ \\
\hline During physiotherapy & $7.9(2.4)$ & $9(3-10)$ \\
\hline After physiotherapy & $6.4(2.5)$ & $6(2-10)$ \\
\hline 30 minutes after physiotherapy & $4.1(3.1)$ & $4(0-8)$ \\
\hline
\end{tabular}

coloured of race and eight were black of race. One subject was Zimbabwean. Ten subjects were burnt by fire, six by hot water and one by hot porridge. The body areas burnt included the face, upper limbs, lower limbs, abdomen, buttocks, back, chest, back of head and trunk. Fourteen of the 17 subjects had second degree burns, while two had a combination of first and second degree burns and one had a combination of second and third degree burns. The median Total Body Surface Area (TBSA) was $22 \%$ (range TBSA of $4 \%$ to $55 \%$ ). The following pharmacological analgesics were administered to the subjects no more than $2 \frac{1}{2}$ hours before commencement of the physiotherapy treatment session: Morphine, Dolorol Forte and Brufen, with dosages varying from $5 \mathrm{mg}$ to $25 \mathrm{mg}$. The body areas treated during the physiotherapy treatment session consisted of: bilateral upper limbs $(n=5)$, bilateral lower limbs $(n=4)$, bilateral hands $(n=1)$, right upper limb $(=5)$, left hand $(n=1)$, and left upper limb $(n=1)$ and the mean duration of the physiotherapy treatment sessions was 17 minutes (range 12 -20 minutes).

\section{Reported pain experienced during physiotherapy treatment session}

Pain scores were obtained before, during, immediately after and 30 minutes after the physiotherapy treatment session. Four pain scores were thus obtained for each subject. The following table (table 1) reports the mean (SD) and median (range) scores for pain before, during, after and 30 minutes after physiotherapy when using pharmacological analgesics only. 
During the physiotherapy session, a median pain score of 9 (range 3-10) was reported, compared to a median score of 5 (range $0-8$ ) before the physiotherapy session, indicating that the majority of the included subjects reported severe pain during the physiotherapy session. The mean pain scores reported during the physiotherapy treatment were significantly higher t-test $(\mathrm{p}=0.006$; MD -3.5; $95 \%$ CI -5.87 to -1.13 ) than the pain scores reported before the commencement of the physiotherapy session. Significantly higher scores t-test $(\mathrm{p}=0.04$; MD -2; 95\% CI -3.89 to -0.11) were also reported after the physiotherapy session compared to before the physiotherapy session. No significant differences t-test ( $\mathrm{p}=0.77$; MD $0.3 ; 95 \% \mathrm{CI}-1.80$ to 2.40 ) were found between before and 30 minutes after the physiotherapy session.

\section{Reported anxiety experienced during physiotherapy treatment session}

Anxiety scores were obtained before, during, immediately after and 30 minutes after the physiotherapy treatment session. Four anxiety scores were thus obtained for each subject. The following table (table 2) illustrates the mean (SD) and median (range) scores for anxiety before, during, after and 30 minutes after physiotherapy when using pharmacological analgesics alone.

During the physiotherapy session, a median anxiety score of 58 (range 27-90) was reported, compared to a median anxiety score of 47 (range 2-78) before the physiotherapy session, indicating that the majority of the included subjects reported higher anxiety levels during than before the physiotherapy session.

Table 2: Anxiety scores (mean and median scores) as reported by subjects before, during and after physiotherapy sessions.

\begin{tabular}{|l|l|l|}
\hline & \multicolumn{2}{|c|}{ Anxiety scores (N=17) } \\
\hline & Mean (SD) & Median (range) \\
\hline Before physiotherapy & $44.8(23.2)$ & $47(2-78)$ \\
\hline During physiotherapy & $60.4(19.5)$ & $58(27-90)$ \\
\hline After physiotherapy & $52.4(22.0)$ & $52(8-88)$ \\
\hline 30 minutes after physiotherapy & $42.5(25.0)$ & $40(0-83)$ \\
\hline
\end{tabular}

sessions are beneficial for their functional recovery, the pain and subsequent anxiety they experience during the physiotherapy rehabilitation may prohibit the patient from complying with the physiotherapist and achieving their goals (Hoffman et al 2000; van Twillert et al 2007). Therefore, in order for physiotherapists to assist burn injury patients in achieving the most optimal functional outcomes in burn injury rehabilitation, adequate management of procedural pain during physiotherapy treatment is required (de Jong et al 2007). It is also essential that a trusting relationship between the physiotherapist and the burn injury victim is maintained (de Jong et al 2007). This can only be achieved if the patient's procedural pain during the physiotherapy sessions is successfully recognized, not underestimated, and appropriately addressed. Since it appears that traditional pharmacologic analgesics are inadequate in successfully managing procedural pain in burn injury patients from developing countries, adjunct therapies are thus warranted.

Non-pharmacologic adjunct therapies usually pose less threat to the burn injury patient than traditional pharmacologic analgesics, as they are associated with less side-effects, are less addictive and less invasive (Hoffman et al 2006). Examples of non-pharmacologic adjunct therapies currently available for burn injury patients are distraction, hypnosis, music therapy and relaxation therapy (Abdi et al 2002; Richardson et al 2009). Previous studies conducted in developed countries amongst burn injury patients have shown that Virtual reality (VR), a distraction technique, in particular is effective in reducing pain and anxiety during physiotherapy treatment sessions and wound dressing changes (Morris et al 2009). The only drawback of an adjunct therapy like VR is that it is expensive, costing in the region of R250 000. In a developing world setting like South Africa, where healthcare budgets are stringent and prioritized to conditions such as HIV/AIDS and tuberculosis (Louw et al 2007), the implementation of effective yet expensive adjunct therapies like VR, may thus not be feasible 
or affordable (Rand et al 2008). Since the prevalence of burns is higher in developing countries (Ahuja et al 2004), future studies should investigate the possibility of implementing less-costly adjunct therapies into developing world clinical burn settings and not simply discard implementation of certain interventions due to cost concerns.

\section{CONCLUSION}

Albeit the results of this study show that burn injury victims in a developing country could benefit from adjunct therapies to the current pain management regimens in place, it does not signify that the adjunct therapies currently utilized in developed countries will be economically feasible. Pragmatic trials investigating the effect of less-cost adjunct interventions for burn injury pain and anxiety in a developing world clinical burn setting are thus warranted.

\section{REFERENCES}

Abdi S and Zhou Y 2002 Management of pain after burn injury. Curr Opin Anaesthesiol 15:563-567

Ahuja R, Bhattacharya S 2004 Burns in the developing world and burn disaster. BMJ 329:447-449
Connor-Ballard P 2009 Understanding and managing burn pain: part 1. AJN 109(4);48-56

De Jong A, Middelkoop E, Faber A, van Loey N 2007 Non-pharmacological nursing interventions for procedural pain relief in adults with burns: a systematic literature review. Burns 33:811-827

Haik J, Tessone A, Nota A, Mendes D, Raz L, Goldan O, Regev E, Winkler E, Mor E, Orenstein A, Hollombe I 2006 The use of video capture virtual reality in burn rehabilitation: the possibilities. Journal of Burn Care and Research 95-197

Hoffman H, Patterson D and Carrougher G 2000 Use of virtual reality for adjunctive treatment of adult burn pain during physical therapy: a controlled study. Clinical Journal of Pain 16(3):244-250

Hoffman H, Seibel E, Richards T, Furness T, Patterson D, Sharar S 2006 Virtual reality helmet display quality influences the magnitude of virtual reality analgesia. The Journal of Pain 7:843-850

Jonsson C, Holmsten A, Dahlström L and Jonsson K 1998 Background pain in burn patients: routine measurement and recording of pain intensity in a burn unit. Burns 24: 448454

Louw Q, Morris L and Grimmer-Somers K 2007 Prevalence of low back pain in Africa: a systematic review. BMC Musculoskeletal Disorders 8:105
Morris LD, Louw QA and Grimmer-Somers K 2009 The effect of Virtual Reality on reducing pain and anxiety in burn injury patients: a systematic review. Clinical Journal of Pain 25(9):815-826

Ptacek J, Patterson D, Montgomery B and Heimbach D 1995 Pain, coping and adjustment in patients with burns: preliminary findings from a prospective study. Journal of pain and symptom management 10(6):446-455

Rand D, Kizony R and Weiss P 2008 The Sony PlayStation II EyeToy: Low cost Virtual Reality for use in Rehabilitation. Journal Neuro logical Physical Therapy 32: 155-163

Richardson P and Mustard L 2009 The management of pain in the burns unit. Burns Nov;35(7):921-36

Taal L and Faber A 1997 The burn specific pain anxiety scale: introduction of a reliable and valid measure. Burns 23(2):147-50

Van Twillert B, Bremer M and Faber A 2007 Computer-generated virtual reality to control pain and anxiety in pediatric and adult burn patients during wound dressing changes Journal of Burn Care and Research 28:1-9 